\title{
Metabolic MRI with hyperpolarized [1-13C]pyruvate separates benign oligemia from infarcting penumbra in porcine stroke
}

Bøgh, Nikolaj; Olin, Rie B.; Hansen, Esben S.S.; Gordon, Jeremy W.; Bech, Sabrina K.; Bertelsen, Lotte B.; Sánchez-Heredia, Juan D.; Blicher, Jakob U.; Østergaard, Leif; Ardenkjær-Larsen, Jan H. Total number of authors: 13

Published in:

Journal of Cerebral Blood Flow and Metabolism

Link to article, DOI:

10.1177/0271678X211018317

Publication date:

2021

Document Version

Peer reviewed version

Link back to DTU Orbit

Citation (APA):

Bøgh, N., Olin, R. B., Hansen, E. S. S., Gordon, J. W., Bech, S. K., Bertelsen, L. B., Sánchez-Heredia, J. D., Blicher, J. U., Østergaard, L., Ardenkjær-Larsen, J. H., Bok, R. A., Vigneron, D. B., \& Laustsen, C. (2021). Metabolic MRI with hyperpolarized [1-13C]pyruvate separates benign oligemia from infarcting penumbra in porcine stroke. Journal of Cerebral Blood Flow and Metabolism, 41(11).

https://doi.org/10.1177/0271678X211018317

\section{General rights}

Copyright and moral rights for the publications made accessible in the public portal are retained by the authors and/or other copyright owners and it is a condition of accessing publications that users recognise and abide by the legal requirements associated with these rights.

- Users may download and print one copy of any publication from the public portal for the purpose of private study or research.

- You may not further distribute the material or use it for any profit-making activity or commercial gain

- You may freely distribute the URL identifying the publication in the public portal 


\section{Metabolic MRI with hyperpolarized $\left[1{ }^{13} \mathrm{C}\right]$ pyruvate separates benign oligemia from infarcting penumbra in porcine stroke}

Nikolaj Bøgh ${ }^{1}$, Rie Beck Olin², Esben Søvsø Szocska Hansen ${ }^{1}$, Jeremy W. Gordon ${ }^{3}$, Sabrina Kahina Bech ${ }^{1}$, Lotte Bonde Bertelsen ${ }^{1}$, Juan Diego Sanchez ${ }^{2}$, Jakob Udby Blicher ${ }^{4,5}$, Leif $\emptyset$ stergaard $^{5}$, Jan Henrik Ardenkjær-Larsen ${ }^{2,6}$, Robert A. Bok ${ }^{3}$, Daniel B. Vigneron ${ }^{3,7}$, Christoffer Laustsen ${ }^{1}$.

1: The MR Research Center, Department of Clinical Medicine, Aarhus University, Aarhus, Denmark.

2: Department of Health Technology, Technical University of Denmark, Kongens Lyngby, Denmark.

3: Department of Radiology and Biomedical Imaging, University of California San Francisco, San Francisco, USA.

4: Department of Neurology, Aarhus University Hospital, Aarhus, Denmark.

5: Center of Functionally Integrative Neuroscience, Aarhus University, Aarhus, Denmark.

6: GE Healthcare, Brøndby, Denmark

7: UC Berkeley-UCSF Graduate Program in Bioengineering, University of California San Francisco and University of California, Berkeley, California, USA.

Please address correspondence to Nikolaj Bøgh at Aarhus University, Palle-Juul Jensens Boulevard 99, 8200 Aarhus N, Denmark, 004522552808 or nikolaj.boegh@clin.au.dk.

Running headline: Penumbral imaging with hyperpolarized MRI

Word count: 3808 


\begin{abstract}
Acute ischemic stroke patients benefit from reperfusion in a short time-window after debut. Later treatment may be indicated if viable brain tissue is demonstrated and this outweighs the inherent risks of late reperfusion. Magnetic resonance imaging (MRI) with hyperpolarized $\left[1{ }^{13} \mathrm{C}\right]$ pyruvate is an emerging technology that directly images metabolism. Here, we investigated its potential to detect viable tissue in ischemic stroke. Stroke was induced in pigs by intracerebral injection of endothelin 1. During ischemia, the rate constant of pyruvate-to-lactate conversion, $k_{\mathrm{PL}}$, was $52 \%$ larger in penumbra and $85 \%$ larger in the infarct compared to the contralateral hemisphere $(P=0.0001)$. Within the penumbra, the $k_{P L}$ was 50 $\%$ higher in the regions that later infarcted compared to non-progressing regions $(P=0.026)$. After reperfusion, measures of pyruvate-to-lactate conversion were slightly decreased in the infarct compared to contralateral. In addition to metabolic imaging, we used hyperpolarized pyruvate for perfusionweighted imaging. This was consistent with conventional imaging for assessment of infarct size and blood flow. Lastly, we confirmed the translatability of simultaneous assessment of metabolism and perfusion with hyperpolarized MRI in healthy volunteers. In conclusion, hyperpolarized $\left[1-{ }^{13} \mathrm{C}\right]$ pyruvate may aid penumbral characterization and increase access to reperfusion therapy for late presenting patients.
\end{abstract}

\title{
Keywords
}

Lactate, Metabolism, MRI, Neuroradiology, Penumbra, Stroke 


\section{Introduction}

Reperfusion with thrombolysis or thrombectomy is the primary treatment of ischemic stroke. The patients are offered reperfusion if treatment can be performed within 4.5 hours. ${ }^{1}$ This time-based approach is often hindered by late hospital arrival or uncertain estimations of onset time. As a result, not all patients are candidates for reperfusion treatment, and even fewer are ultimately treated. ${ }^{2}$ There is an urgent need for personalizing stroke care by basing therapy decisions on the presence of viable brain tissue in each individual patient rather than a short time-window after symptom onset.

Magnetic resonance imaging (MRI) may more precisely pinpoint the patients that will benefit from reperfusion. Perfusion-weighted imaging promises identification of salvageable penumbra by detecting the diffusion-perfusion mismatch. Hereby, it is possible to extend the window of treatment to 16 hours after symptom onset. ${ }^{3}$ But perfusion-weighted imaging struggles to distinguish areas of benign oligemia from true penumbral tissue, and perfusion-based ischemic thresholds are poorly defined. ${ }^{4-6}$ Patients with similar diffusion-perfusion mismatches can have vastly different outcomes upon reperfusion. ${ }^{7}$ This reflects that the salvageability of the penumbra is not entirely captured with perfusion-weighted imaging. For these reasons, the technique has not had the anticipated clinical impact, and decisions outside of the timewindow are currently based on mismatches between diffusion weighted imaging and symptoms or edema-sensitive anatomical MRI. ${ }^{8,9}$ Nevertheless, penumbral imaging remains compelling for individualizing care. One opportunity may be to directly image the metabolic crisis that defines an ischemic stroke.

Currently under clinical translation, hyperpolarized pyruvate MRI provides unpreceded metabolic information in a rapid one-minute acquisition. ${ }^{10}$ With hyperpolarization, the MR signal of $\left[1-{ }^{13} \mathrm{C}\right]$ pyruvate is augmented $>10,000$-fold before it is injected and the pyruvate and its metabolites - lactate and 
bicarbonate - are detected. They are quantified using models or as ratios which represent a compound of processes, including pyruvate or lactate dehydrogenase (PDH and LDH) flux and metabolite pool size. As a marker sensitive to hypoxia, the exchange of pyruvate to lactate may aid characterization of stroke metabolism. In addition to metabolic imaging, it is possible to use the hyperpolarized tracers such as urea and pyruvate as contrast agents for perfusion-weighted imaging. ${ }^{11-13}$ To date, two studies have investigated hyperpolarized MRI in rodent models of ischemic stroke. They suggest that conversion between pyruvate and lactate increases shortly after reperfusion and then normalizes before it increases again after several hours. ${ }^{14,15}$ However, no studies have evaluated hyperpolarized MRI during ischemia, and none have used hyperpolarized pyruvate for perfusion-weighted imaging in stroke.

We hypothesized that MRI with hyperpolarized $\left[1-{ }^{13} \mathrm{C}\right]$ pyruvate can depict the metabolic consequences of ischemic stroke. Further, we hypothesized that it allows estimation of perfusion, yielding assessment of metabolism and perfusion in one scan. Accordingly, we performed hyperpolarized $\left[1-{ }^{13} \mathrm{C}\right]$ pyruvate MRI before and after reperfusion in an endothelin 1 (ET-1) based model in pigs. ${ }^{16}$ This was supported with tissue analysis and conventional imaging. Lastly, we performed hyperpolarized MRI in healthy human volunteers to assess the translatability of simultaneous metabolism and perfusion imaging.

\section{Material and Methods}

\section{Study overview}

This paper contains data from preclinical work and healthy humans. We induced ischemic stroke in pigs by intracerebral injection of ET-1. To characterize reperfusion after ET-1, we assessed cerebral blood flow (CBF) every 20 minutes for 4 hours $(n=3)$. We ascertained that the brain was reperfused from $\sim 1$ to 3.5 hours. Then, we performed hyperpolarized $\left[1-{ }^{13} \mathrm{C}\right]$ pyruvate $\mathrm{MRI}(\mathrm{n}=7)$ before and after reperfusion 
(Figure 1a). The experiments were approved by the Danish Animal Inspectorate (protocol 2018-15-020101411), conforms to European legislation (Directive 2010/63/EU) and are reported following the ARRIVE guidelines. The human study was approved by the institutional review board at the University of California, San Francisco and followed the World Medical Association Declaration of Helsinki. Three healthy volunteers (male, aged 29 to 38) were included after written informed content. One was imaged twice, yielding four datasets. An expanded Material and Methods section and an overview of missing preclinical data (Table S1) are provided in the supplement.

\section{Pig stroke model}

Ten Female Danish landrace pigs (36-44 kg, 12 weeks of age) were included. The pigs were anaesthetized with propofol ( $3 \mathrm{mg} / \mathrm{kg}$ followed by $4-8 \mathrm{mg} / \mathrm{kg} / \mathrm{h} \mathrm{IV}$ ) and fentanyl $(0.01 \mathrm{mg} / \mathrm{kg}$ followed by $0.035 \mathrm{mg} / \mathrm{kg} / \mathrm{h}$ IV) and ventilated with $30 \%$ oxygen for a target arterial $\mathrm{CO}_{2}$ of 5.0 to $5.5 \mathrm{kPa}$. Catheters for monitoring, blood sampling and injections were placed in the femoral vein, the femoral artery and the internal jugular vein ipsilateral to the stroke. A $2 \mathrm{~cm}$ burr hole was drilled $1.5 \mathrm{~cm}$ from the midline caudal to the orbit. A thin needle (30 gauge) was inserted $1 \mathrm{~cm}$ through the intact dura. ET-1 (30 $\mu \mathrm{g}$, Sigma-Aldrich) was dissolved in saline $(200 \mu \mathrm{l})$ and injected over 10 minutes.

\section{Magnetic resonance imaging}

MRI was performed on clinical 3T systems (Discovery MR750, GE Healthcare). Proton images were obtained with standard, commercial coils. The pigs and volunteers underwent routine T1-weighted and T2-weighted imaging. CBF was assessed with Arterial Spin Labeling. In addition to these, the pigs underwent diffusion weighted imaging, single-voxel spectroscopy, parametric mapping, sodium imaging 
and dynamic susceptibility contrast (DSC) imaging during injection of a gadolinium-based contrast agent (10 ml flushed with $20 \mathrm{ml}$ saline, $\sim 5 \mathrm{ml} / \mathrm{s}$, GE Healthcare). This was omitted at the 1-hour timepoint to avoid effects on the hyperpolarized imaging. Detailed scan parameters are available in the supplement. For concurrent perfusion and metabolic imaging, we performed hyperpolarized $\left[1-{ }^{13} \mathrm{C}\right]$ pyruvate MRI. After hyperpolarization (see below), the $\left[1-^{13} \mathrm{C}\right]$ pyruvate solution was injected intravenously at $\sim 5 \mathrm{ml} / \mathrm{s}(\sim 0.875$ $\mathrm{ml} / \mathrm{kg}$ for pigs, $0.43 \mathrm{ml} / \mathrm{kg}$ for volunteers) and flushed with $20 \mathrm{ml}$ saline. Spectral-spatial imaging was started to capture the temporal dynamics of the pyruvate, lactate and bicarbonate resonances. For the preclinical studies, we used a fully-sampled adaptation of a previously described sequence employing a stack-of-spirals readout $\left(6^{\circ} / 37^{\circ} / 37^{\circ}\right.$ flip angles on pyruvate/lactate/bicarbonate).$^{17}$ It covered the entire brain with a resolution of $1 \times 1 \times 1.5 \mathrm{~cm}^{3}$. The temporal resolution was $960 \mathrm{~ms}$ for pyruvate and $2880 \mathrm{~ms}$ for lactate and bicarbonate. We used a custom-made 14-channel receive coil with a volume transmit (RAPID Biomedical, Germany). For the human studies, we used a head transceiver coil and a multi-slice echo planar imaging sequence $\left(10^{\circ} / 40^{\circ} / 40^{\circ}\right.$ flip angles on pyruvate/lactate/bicarbonate) ${ }^{18}$ Temporal resolution was 3 seconds and spatial resolution was $1.5 \times 1.5 \mathrm{~cm}^{2}$. To cover the entire brain, 8 slices of $1.5 \mathrm{~cm}$ were acquired.

\section{Hyperpolarization of pyruvate}

Dynamic nuclear polarization of $\left[1{ }^{13} \mathrm{C}\right]$ pyruvic acid (Sigma-Aldrich) was performed in a commercial system (SpinAligner, Polarize or SPINLab, GE Healthcare). ${ }^{19}$ The sample was polarized for over 2 hours, typically yielding polarization levels of 30-40\%. After hyperpolarization, the sample was dissolved and neutralized to a final concentration of $250 \mathrm{mM}\left[1{ }^{-13} \mathrm{C}\right]$ pyruvate. Time to injection was $\sim 55 \mathrm{~s}$ for human examinations and $~ 10$ s for preclinical examinations. 
Image processing and analyses

The hyperpolarized $\left[1{ }^{13} \mathrm{C}\right]$ pyruvate data were reconstructed in MATLAB (R2019b, MathWorks). A twoside metabolic exchange kinetic model was applied for estimation of the first order apparent rate constant of exchange from pyruvate to lactate $\left(k_{\mathrm{PL}}\right) \cdot{ }^{20}$ Furthermore, ratios of summed signal of pyruvate to lactate and bicarbonate were calculated.

Perfusion-weighted imaging was analyzed using model-free deconvolution. For hyperpolarized MRI, we used the pyruvate signal timeseries. Two to four voxels were automatically selected for fitting of an arterial input function. ${ }^{21}$ Mean transit time (MTT) and relative CBF (rCBF) were computed using block-circulant singular value decomposition. Hyperpolarized pyruvate perfusion estimates were corrected for depolarization. ${ }^{11}$

All images were analyzed using the imaging processing software Horos (The Horos Project, Horosproject.org). Apparent diffusion coefficient (ADC) and CBF maps were automatically segmented using thresholding. The perfusion defect was defined as CBF below $25 \mathrm{ml} / 100 \mathrm{ml} / \mathrm{min} .{ }^{4}$ Infarct core was defined as an $A D C$ below $620 \times 10^{-6} \mathrm{~mm}^{2} / \mathrm{s}^{22}$ The penumbra was the mismatch between the two. It disappeared with reperfusion and was only analyzed for the ischemia timepoint. The remaining images were segmented manually. Blinding was impossible due to the nature of the data.

\section{Biochemistry}

To validate the arterial input function of the hyperpolarized MRI, arterial blood samples of $\sim 3 \mathrm{ml}$ were drawn every two seconds during the pyruvate injection $(n=3)$ and analyzed using a blood gas analyzer (ABL90 FLEX PLUS, Radiometer Copenhagen, Denmark). After sacrifice, biopsies were obtained for 
analyses of lactate concentration and enzyme activities. Plasma and biopsies were analyzed using colorimetric assays (Sigma-Aldrich).

\section{Statistics}

Data are presented as mean \pm standard deviation (SD). Normality was assessed using quantile-quantile plots. Testing was performed with linear mixed-effect models ${ }^{23}$ or Friedman's test. Effect estimates are reported with $95 \%$ confidence intervals $(95 \% \mathrm{Cl})$. All models are reported in Table S2. Bland and Altman's approach was used for repeated-measures correlation assessment. ${ }^{24} \mathrm{~A}$ local regression curve was added to Figures 1b, 4a and S1a to ease interpretation. Statistics were performed in R (R Core Team 2014, Rproject.org).

\section{Results}

Characteristics of the porcine stroke

In the pig stroke model, ET-1 caused a perfusion defect of $8.8 \pm 6.4 \mathrm{ml}$ with a diffusion-perfusion mismatch of $4.98 \pm 5.73 \mathrm{ml}$ at 1 hour after injection. The perfusion defect gradually shrank to $2.2 \pm 3.9 \mathrm{ml}$ at 3.5 hours after ET-1, while CBF doubled outside this defect (Figures $1 \mathrm{~b}$ and S1). The hypoperfusion caused an infarct that grew from $3.8 \pm 1.7 \mathrm{ml}$ to $10.7 \pm 6.2 \mathrm{ml}(6.8 \mathrm{ml}$ growth; $95 \% \mathrm{Cl}, 3$ to $10.8 \mathrm{ml}$; Figure $1 \mathrm{c})$. [insert Figure 1.]

To facilitate interpretation of the hyperpolarized MRI, we performed an MRI protocol for tissue characterization at 1 and 3.5 hours and biochemical analyses of biopsies obtained after sacrifice. As a measure of neural viability, the $\mathrm{N}$-acetylaspartate-to-creatine ratio measured with spectroscopy decreased $(-0.39 ; 95 \% \mathrm{Cl},-0.24$ to -0.54 ; Figure $2 \mathrm{a})$. The lactate-to-creatine ratio showed an insignificant 
tendency to increase $(0.89 ; 95 \% \mathrm{Cl},-0.06$ to 1.81$)$. In biopsies (Figure $2 \mathrm{~b}$ ), an increased lactate concentration was found ipsilateral to the infarct. Before normalization to total protein, LDH and PDH activities were $2.34(95 \% \mathrm{Cl}, 0.3$ to 4.4$)$ and $2.96(95 \% \mathrm{Cl}, 0.02$ to 5.9$)$ units lower than contralateral. This difference disappeared with normalization. Using parametric MRI (Figure S2), we found no changes in T1 relaxation time with region or injection of gadolinium-based contrast. T2 was longer after reperfusion and R2* was increased during ischemia in the infarct, suggesting edema and ischemia, respectively. With sodium MRI, we observed an initial fall in sodium signal compared to contralateral brain (extrapolated to $\sim 90 \%$ with a linear fit), which then increased 0.0009 percent points per minute $(95 \% \mathrm{Cl}, 0.0006$ to 0.001$)$. [insert Figure 2.]

To monitor the systemic metabolism and cardiovascular state, we recorded heart rate, blood pressure and arterial gasses, glucose and lactate throughout the experiment (Figure S3a). Of these, blood glucose and lactate increased slightly with time. Glucose remained within normal, while blood lactate increased to above $2 \mathrm{mmol}$. The artery to jugular vein difference of lactate, oxygen and carbon dioxide did not change, while the glucose difference decreased towards zero (Figure S3b).

Hyperpolarized MRI for metabolic assessment of ischemic stroke

We performed hyperpolarized $\left[1-{ }^{13} \mathrm{C}\right]$ pyruvate MRI during ischemia (before 1 hour) and after reperfusion (after 3.5 hours). Times to scan were $51.8 \pm 12.4$ and $218 \pm 43.4$ minutes. The signal-to-noise ratios of temporally summed data were $60.2 \pm 24.3$ for pyruvate, $23 \pm 11.2$ for lactate and $6.2 \pm 1.3$ for bicarbonate. Examples of raw data and a map of model-based lactate-to-pyruvate exchange $\left(k_{\mathrm{PL}}\right)$ are presented in Figures $3 a, 3 b$ and S4. During ischemia, $k_{\mathrm{PL}}$ was $0.0125 \mathrm{~s}^{-1}(95 \% \mathrm{Cl}, 0.01$ to 0.0149$)$ higher in the infarct and $0.0077 \mathrm{~s}^{-1}(95 \% \mathrm{Cl}, 0.0052$ to 0.0101$)$ higher in the penumbra compared to the contralateral hemisphere 
$\left(0.0147 \pm 0.0027 \mathrm{~s}^{-1}\right)$. After reperfusion, infarct $k_{\mathrm{PL}}$ was decreased by $0.0021 \mathrm{~s}^{-1}(95 \% \mathrm{Cl}, 0.0004$ to 0.0037$)$ compared to contralateral (Figure 3c). We found comparable results using model-free quantification (Figure 3d).

[insert Figure 3.]

Perfusion-weighted imaging of porcine stroke with hyperpolarized pyruvate

We estimated MTT and rCBF from the hyperpolarized pyruvate data (Figures $4 a+b)$. The arterial input functions from imaging and arterial sampling corresponded well in two pigs and fairly in the last (Figure S5). The baseline pyruvate concentration was $57 \pm 14 \mathrm{ng} / \mathrm{ul}$, which increased to $7054 \pm 2368 \mathrm{ng} / \mathrm{ul}$ at the peak. Hyperpolarized pyruvate-derived rCBF values were slightly larger compared to gadolinium DSC imaging ( $1.27 \pm 0.49$ vs $0.96 \pm 0.28)$, but unskewed at low and high rCBF and with decent agreement (Figure 4c). MTT was considerably longer when calculated from hyperpolarized pyruvate $(25.3 \pm 9.4$ s vs $2.8 \pm 0.7$ s). Bland-Altman plotting revealed a bias towards relatively longer MTTs with lower perfusion. When comparing with arterial spin labeling MRI (Figure 4d), we found that CBF correlated with both rCBF $(r=$ $0.74 ; 95 \% \mathrm{Cl}, 0.46$ to 0.88$)$ and $\mathrm{MTT}(r=-0,76 ; 95 \% \mathrm{Cl},-0.9$ to -0.5$)$. Further, perfusion defect size showed good agreement (Figure 4e).

[insert Figure 4.]

Penumbral characterization with hyperpolarized pyruvate MRI

To evaluate if hyperpolarized MRI may predict penumbral outcome, we compared the parts of the penumbra that infarcted between 1 and 3.5 hours to the parts that did not (Figure 5). The $k_{\mathrm{PL}}$ was 0.0254 $\pm 0.0064 \mathrm{~s}^{-1}$ in the regions that infarcted, while it was $0.0169 \pm 0.0053 \mathrm{~s}^{-1}$ in the regions that did not 
(difference $0.0085 \mathrm{~s}^{-1} ; 95 \% \mathrm{Cl}, 0.004$ to 0.013$)$. The lactate/pyruvate ratio was increased by $0.17(95 \% \mathrm{Cl}$, 0.08 to 0.27$)$. Pyruvate MTT was longer in the progressing regions, while rCBF trended to be lower, albeit insignificantly. ASL-derived CBF and ADC values were comparable between infarcting and non-infarcting penumbra (CBF difference $-2.3 \mathrm{ml} / 100 \mathrm{ml} / \mathrm{min} ; 95 \% \mathrm{Cl},-6.1$ to 1.5 and ADC difference $-33.5 \times 10^{6} \mathrm{~mm}^{2} / \mathrm{s}$; $95 \% \mathrm{Cl},-71.1$ to 4.2$)$

[insert Figure 5.]

Imaging human metabolism and perfusion with hyperpolarized MRI

To evaluate the translational potential of simultaneous assessment of metabolism and perfusion, we performed hyperpolarized MRI in healthy human volunteers (Figure 6a). We found $k_{\mathrm{PL}}$ to be $0.0237 \pm$ $0.0015 \mathrm{~s}^{-1}$ in cortex and $0.0214 \pm 0.0026 \mathrm{~s}^{-1}$ in deep white matter (Figure 6b). Perfusion-weighted quantification (Figure $6 c$ ) of $\mathrm{rCBF}$ and MTT correlated with ASL derived CBF $(r=0.88 ; 95 \% \mathrm{Cl}, 0.67$ to 0.96 and $r=-0.85 ; 95 \% \mathrm{Cl},-0.95$ to -0.61$)$.

[insert Figure 6.]

\section{Discussion}

We examined the potential of hyperpolarized $\left[1-{ }^{13} \mathrm{C}\right]$ pyruvate MRI for imaging of metabolism and perfusion in acute ischemic stroke. We found increased pyruvate-to-lactate exchange in ischemic regions. The penumbra that infarcted had increased exchange to lactate compared to regions of benign oligemia. Further, perfusion estimation using the hyperpolarized pyruvate data agreed well with perfusion measures derived from arterial spin labeling in both pigs and humans. 
We chose to use the porcine ET-1 model of acute ischemic stroke. This approach produced an infarct core with surrounding penumbra, lactate accumulation and neuronal loss, followed by gradual reperfusion. Even though widely used in rodent models, one limitation of ET-1 is its potential non-ischemic effects through receptors on some brain cells. ${ }^{25}$ However, endovascular models are impossible due to the vascular anatomy of the pig, and established models involve extensive surgery. The ET-1 model is a much less invasive choice. ${ }^{26}$ Further, the model eased repetition of imaging during ischemia and after reperfusion. The pig has some advantages over rodents. First, its size allows use of clinical scanners. Second, its brain is gyrencephalic, and third, it has a high white-to-gray matter ratio, making its metabolic demands more similar to the human brain. This makes the pig a good model of human stroke in imaging studies. ${ }^{26}$

Hyperpolarized $\left[1-{ }^{13} \mathrm{C}\right]$ pyruvate MRI yields direct metabolic information undetectable by current imaging. Previous efforts have found increased exchange between pyruvate and lactate in stroke. ${ }^{14,15}$ However, they did not differ between the infarct core and the penumbra. Here, we performed imaging during ischemia, allowing us to probe penumbral metabolism. Our findings correspond to microdialysis studies, where the lactate/pyruvate ratio is a marker of ischemia and deterioration. ${ }^{27}$ Hyperpolarized MRI could be a viable, less invasive and whole-brain covering alternative to microdialysis, which is rarely used in stroke. Surprisingly, we found increased bicarbonate-to-pyruvate ratios in the ischemic areas. We speculate that decreased delivery and changes in the extraction fraction of pyruvate may partly explain this. However, that finding should be interpreted with care until evaluated by other studies due to relatively low signal. Similarly to Hyacinthe and colleagues, we found that hyperpolarized pyruvate-tolactate exchange decreased below normal after reperfusion despite high tissue content of lactate. ${ }^{15}$ This is likely driven by increased pyruvate delivery from hyperperfusion in combination with cell death. In contradiction, a previous study found increased lactate production 18 hours post-reperfusion. ${ }^{14}$ Numerous factors may cause a secondary rise in lactate production, including inflammation, ${ }^{28}$ which is 
detectable with hyperpolarized MRI. ${ }^{29}$ Hereby, the technology may find use in the sub-acute setting, providing monitoring of novel immunomodulatory and neuroprotective treatments. ${ }^{30}$ However, as we expect little inflammation in the short duration of our experiments, the data presented here are unable to confirm that hypothesis.

Perfusion-weighted imaging with hyperpolarized MRI was first suggested by Johansson and colleagues. ${ }^{11}$ It has been shown to provide reasonably accurate perfusion estimates in kidney injury, ${ }_{1}^{13}$ healthy hearts ${ }^{31}$ and tumors. ${ }^{12}$ But using pyruvate violates the assumption that the tracer is not metabolized. Actually, the arterial-venous differences of oxygen and glucose could suggest that pyruvate could partly have replaced glucose in our experiment. However, we found that the blood concentration of pyruvate increased more than 100 -fold to $~ 80 \mathrm{mM}$ during the first pass of the bolus, which is used for perfusion-weighted imaging. Even with the dose used in humans, we expect relatively little metabolism of pyruvate due to saturation of blood-brain barrier transport, which has a $K_{\mathrm{m}}$ of 6 to $1.5 \mathrm{mM}$ in young and adult rats, respectively. ${ }^{32}$ But, however little, our perfusion estimates still carry a metabolic component. ${ }^{33}$ In our comparison between DSC, ASL and hyperpolarized pyruvate MRI, the different metabolic rates of ischemic and healthy brain seems to introduce a bias towards underestimation or overestimation of perfusion, respectively. Of note, this could also be influenced by variation in relaxation properties, which were not considered in our density-weighted data. Unsurprisingly, we found prolonged MTTs comparing hyperpolarized pyruvate to gadolinium. This is likely the result of differences in transport kinetics and bolus size. To further improve perfusion estimates, one could co-polarize pyruvate with urea, which is not metabolized. ${ }^{12}$ Also, this could elucidate potential effects from relaxation mechanisms. ${ }^{34}$ However, hyperpolarized urea is currently not approved for human use. Our data show that hyperpolarized pyruvate perfusion-weighted imaging is a simple alternative that is translatable to humans. It provides good estimation of infarct size and relative perfusion quantification while offering added metabolic insight within a single injection. 
By direct imaging of the metabolic effects of hypoxia, hyperpolarized pyruvate MRI could aid penumbral characterization. Current MRI detects the perfusion-diffusion mismatch, which is used as a surrogate of salvageable penumbra. ${ }^{5}$ But the potential of perfusion-weighted imaging has not translated fully into clinical impact. One explanation may be that it fails to consider microvascular dysfunction or uncoupling of perfusion and metabolism. Heterogeneity or extreme homogenization of capillary transit times may impair oxygen extraction at a given CBF, explaining the heterogeneity of penumbral outcome and difficulties in defining ischemic thresholds. ${ }^{35,36}$ Whether of microvascular or macrovascular cause, direct assessment of the metabolic consequences of ischemia may allow differentiation of benign oligemia from truly threatened tissue. We found penumbral metabolic heterogeneity in the form of elevated pyruvateto-lactate exchange and decreased pyruvate perfusion in the regions that progressed to infarct. Of note, careful interpretation of the metabolic estimates is warranted, as conversion of lactate to pyruvate is neglected in the kinetic model, and as they are likely affected by perfusion and membrane transport. ${ }^{20,37,38}$ Thus, the quantities should not be interpreted solely as a change in LDH flux per se, but rather as compound measures. Interestingly, similar to the findings presented here, a clinical microdialysis study have found an increased lactate/pyruvate ratio in the tissue that progresses to infarct. ${ }^{39}$ This suggests that hyperpolarized pyruvate MRI detects larger pyruvate-to-lactate exchange in tissue with a worse prognosis. Future studies should evaluate if that effect is large enough, consistent and with sufficient predictive power to be clinically relevant. They may also investigate the potential of complementing current advanced MRI with hyperpolarized MRI, as it can be performed in extension to current MRI protocols. If our findings are confirmed, the technology may aid in further individualizing patient selection beyond the time-based approach.

MRI with hyperpolarized pyruvate is translating to research in volunteers and patients. ${ }^{18,40,41}$ We found metabolic estimates comparable to previous studies, and our data validate hyperpolarized $\left[1-{ }^{13} \mathrm{C}\right]$ pyruvate 
for perfusion-weighted imaging in humans. Hyperpolarized MRI has been applied in volunteers and patients with chronic illness, but stroke is an acute disease where millions of neurons and synapses die every minute. Any addition to MRI protocols must be robust and worthwhile. Hyperpolarized MRI offers this potential, but the technology is not ready yet, as the process of hyperpolarization is time-consuming and current hardware lacks reliability. This may be solved by alternative means of hyperpolarization or by separating hyperpolarization from dissolution. ${ }^{42-44}$ In addition to hardware, the imaging sequences may be optimized. Current clinical approaches offer spatial resolutions of $1.5 \times 1.5 \times 1.5 \mathrm{~cm}^{3}$. Better resolution is warranted for detection of small infarcts and infarcts in proximity to vessels. Refocused imaging, variable flip-angle schemes or acceleration techniques may offer improvements..$^{45}$ Of particular interest here, variable-resolution imaging may improve the resolution on pyruvate for improved perfusion estimation without sacrifice. ${ }^{46}$ Even though partial volume effects and logistic issues currently hinder clinical use, the promise of hyperpolarized MRI could foster solutions.

In conclusion, hyperpolarized $\left[1-{ }^{13} \mathrm{C}\right]$ pyruvate is capable of imaging ischemia and impaired perfusion in a pig model of stroke. Higher pyruvate-to-lactate exchange was found in the penumbral areas that progressed to infarct, suggesting a predictive potential for metabolic penumbral characterization that may complement current MRI protocols aiming to advance treatment personalization. Further, the technology is capable of providing perfusion estimates with the metabolic assessment.

\section{Acknowledgements}

We would like to acknowledge Duy Anh Dang and Mette Dalgaard for their laboratory assistance. 


\section{Author contribution statement}

The project was designed by NB, ESSH and CL. Experimental work and data acquisition was performed by NB, RBO, ESSH, JWG, SKB, LBB, JDS, RAB and DBV. Data were analyzed and interpreted by NB, RBO, ESSH, JWG, JUB, L $\varnothing$, JHAL, DBV and CL. All authors revised and approved the manuscript.

\section{Conflicts of interest}

The Authors declare that there are no conflicts of interest

\section{Funding}

The author(s) disclosed receipt of the following financial support for the research, authorship, and/or publication of this article: This work was funded by the Lundbeck Foundation [grant number: R272-20174023]. Human studies were performed at the UCSF Hyperpolarized MRI Technology Resource Center with funding from NIH P41EBEB013598 and the UCSF NICO project.

\section{Supplementary information}

Supplemental material for this paper can be found at the journal website: http://journals.sagepub.com/home/jcb 


\section{References}

1 Campbell BCV, De Silva DA, Macleod MR, Coutts SB, Schwamm LH, Davis SM et al. Ischaemic stroke. Nat Rev Primer 2019; 5: 70.

2 Fang MC, Cutler DM, Rosen AB. Trends in thrombolytic use for ischemic stroke in the United States. J Hosp Med 2010; 5: 406-409.

3 Albers GW, Marks MP, Kemp S, Christensen S, Tsai JP, Ortega-Gutierrez S et al. Thrombectomy for Stroke at 6 to 16 Hours with Selection by Perfusion Imaging. N Engl J Med 2018; 378: 708-718.

4

Fisher M, Bastan B. Identifying and utilizing the ischemic penumbra. Neurology 2012; 79: S79-S85.

Heiss W-D. The concept of the penumbra: can it be translated to stroke management? Int J Stroke Off J Int Stroke Soc 2010; 5: 290-295.

Demeestere J, Wouters A, Christensen S, Lemmens R, Lansberg MG. Review of Perfusion Imaging in Acute Ischemic Stroke: From Time to Tissue. Stroke 2020; 51: 1017-1024.

Mundiyanapurath S, Diatschuk S, Loebel S, Pfaff J, Pham M, Möhlenbruch MA et al. Outcome of patients with proximal vessel occlusion of the anterior circulation and DWI-PWI mismatch is time-dependent. Eur J Radiol 2017; 91: 82-87.

Thomalla G, Simonsen CZ, Boutitie F, Andersen G, Berthezene Y, Cheng B et al. MRIGuided Thrombolysis for Stroke with Unknown Time of Onset. N Engl J Med 2018; 379: 611-622.

Nogueira RG, Jadhav AP, Haussen DC, Bonafe A, Budzik RF, Bhuva P et al. Thrombectomy 6 to 24 Hours after Stroke with a Mismatch between Deficit and Infarct. N Engl J Med 2018; 378: 11-21. 10 Grist JT, Miller JJ, Zaccagna F, McLean MA, Riemer F, Matys T et al. Hyperpolarized ${ }^{13} \mathrm{C}$ MRI: A novel approach for probing cerebral metabolism in health and neurological disease. J Cereb Blood Flow Metab 2020; 40: 1137-1147. 
perfusion and metabolism using multiple hyperpolarized ${ }^{13} \mathrm{C}$ compounds: HP001, pyruvate and urea. Magn Reson Imaging 2012; 30: 305-11.

Mariager CO, Lindhardt J, Nielsen PM, Schulte RF, Ringgaard S, Laustsen C. Fractional Perfusion: A Simple Semi-Parametric Measure for Hyperpolarized ${ }^{13} \mathrm{C}$ MR. IEEE Trans Radiat Plasma Med Sci 2019; 3: 523-527. Magnetic Resonance Imaging Can Detect Metabolic Changes Characteristic of Penumbra in Ischemic Stroke. Tomography 2017; 3: 67-73. of hyperpolarised $\left[1-{ }^{13} \mathrm{C}\right]$ L-lactate as a neuroprotectant metabolic biosensor for stroke. Sci Rep 2020; 10 : 5507.

133: $245-261$. dimensional accelerated acquisition for hyperpolarized $13 \mathrm{C}$ MR with blipped stack-of-spirals and conjugate-gradient SENSE. Magn Reson Med 2020; 84: 519-534. Carbon-13 EPI for hyperpolarized MR molecular imaging of prostate and brain cancer patients. Magn Reson Med 2019; 81: 2702-2709. Increase in signal-to-noise ratio of $>10,000$ times in liquid-state NMR. Proc Natl Acad Sci U A 2003; 100: 10158-63. Hyperpolarized Carbon-13 Pyruvate Metabolism in the Human Brain. IEEE Trans Med Imaging 2019; 39: 320-327.

21

Peruzzo D, Bertoldo A, Zanderigo F, Cobelli C. Automatic selection of arterial input function on dynamic contrast-enhanced MR images. Comput Methods Programs Biomed 2011; 104: e148157. 

Apparent diffusion coefficient threshold for delineation of ischemic core. Int J Stroke Off J Int Stroke Soc 2015; 10: 348-353.

Bates D, Mächler M, Bolker B, Walker S. Fitting Linear Mixed-Effects Models Using Ime4. J Stat Softw 2015; 67: 1-48. astrocytic endothelin-1. Prog Neurobiol 2016; 144: 88-102.

Kaiser E, West F. Large animal ischemic stroke models: replicating human stroke pathophysiology. Neural Regen Res 2020; 15: 1377-1387. Brain Injury: The Current Status and Potential Future for Cerebral Microdialysis. J Neurotrauma 2005; 22: $3-41$. foe for ischemic stroke. J Neuroinflammation 2019; 16: 142. imaging of Traumatic Brain Injury. Sci Rep 2017; 7: 17525. treatment. Nat Rev Neurol 2019; 15: 473-481. myocardial first-pass cardiovascular magnetic resonance perfusion imaging using hyperpolarized $\left[1-{ }^{13} \mathrm{C}\right]$ pyruvate. J Cardiovasc Magn Reson 2018; 20: 73. Brain Barrier Transport of Pyruvate, Lactate and Glucose in Suckling, Weanling and Adult Rats. J Neurochem 1979; 33: 439-445.

33 Mikkelsen EFR, Mariager C $\varnothing$, Nørlinger T, Qi H, Schulte RF, Jakobsen S et al. Hyperpolarized $\left[1-^{13} \mathrm{C}\right]$-acetate Renal Metabolic Clearance Rate Mapping. Sci Rep 2017; 7: 16002. 
${ }^{13} \mathrm{C}$ urea relaxation mechanism reveals renal changes in diabetic nephropathy. Magn Reson Med 2016; 75: 515-518. Role of the Cerebral Capillaries in Acute Ischemic Stroke: The Extended Penumbra Model. J Cereb Blood Flow Metab 2013; 33: 635-648. time homogenization in ischemic stroke - A novel biomarker of penumbral microvascular failure? J Cereb Blood Flow Metab 2018; 38: 2006-2020. Modeling and Constrained Reconstruction of Hyperpolarized $\left[1-{ }^{13} \mathrm{C}\right]$-Pyruvate Offers Improved Metabolic Imaging of Tumors. Cancer Res 2015; 75: 4708-4717.

$\left.{ }^{13} \mathrm{C}\right]$ pyruvate-to-[1- $\left.{ }^{13} \mathrm{C}\right]$ lactate conversion is rate-limited by monocarboxylate transporter-1 in the plasma membrane. Proc Natl Acad Sci 2020; : 202003537. and Metabolic Changes in Association With Infarct Progression. Stroke 2014; 45: 1183-1185.

Autry AW, Gordon JW, Chen H-Y, LaFontaine M, Bok R, Van Criekinge M et al. Characterization of serial hyperpolarized ${ }^{13} \mathrm{C}$ metabolic imaging in patients with glioma. Neurolmage Clin 2020; 27: 102323. human brain metabolism using hyperpolarized $\left[1-{ }^{13} \mathrm{C}\right]$ pyruvate and magnetic resonance imaging. Neuroimage 2019; 189: 171-179. metabolites. Nat Commun 2017; 8: 1-7. induced radicals following dynamic nuclear polarization to produce transportable frozen hyperpolarized ${ }^{13}$ C-substrates. Nat Commun 2017; 8: 15757. 
imaging of brute-force ${ }^{13} \mathrm{C}$ hyperpolarization. J Magn Reson San Diego Calif 1997 2015; 261: 87-94.

45

Gordon JW, Chen HY, Dwork N, Tang S, Larson PEZ. Fast Imaging for Hyperpolarized MR Metabolic Imaging. J Magn Reson Imaging 2020. doi:10/ggsdsc.

46

Gordon JW, Autry AW, Tang S, Graham JY, Bok RA, Zhu X et al. A variable resolution

approach for improved acquisition of hyperpolarized ${ }^{13} \mathrm{C}$ metabolic MRI. Magn Reson Med 2020. doi:10/gg55n9. 


\section{Figure legends}

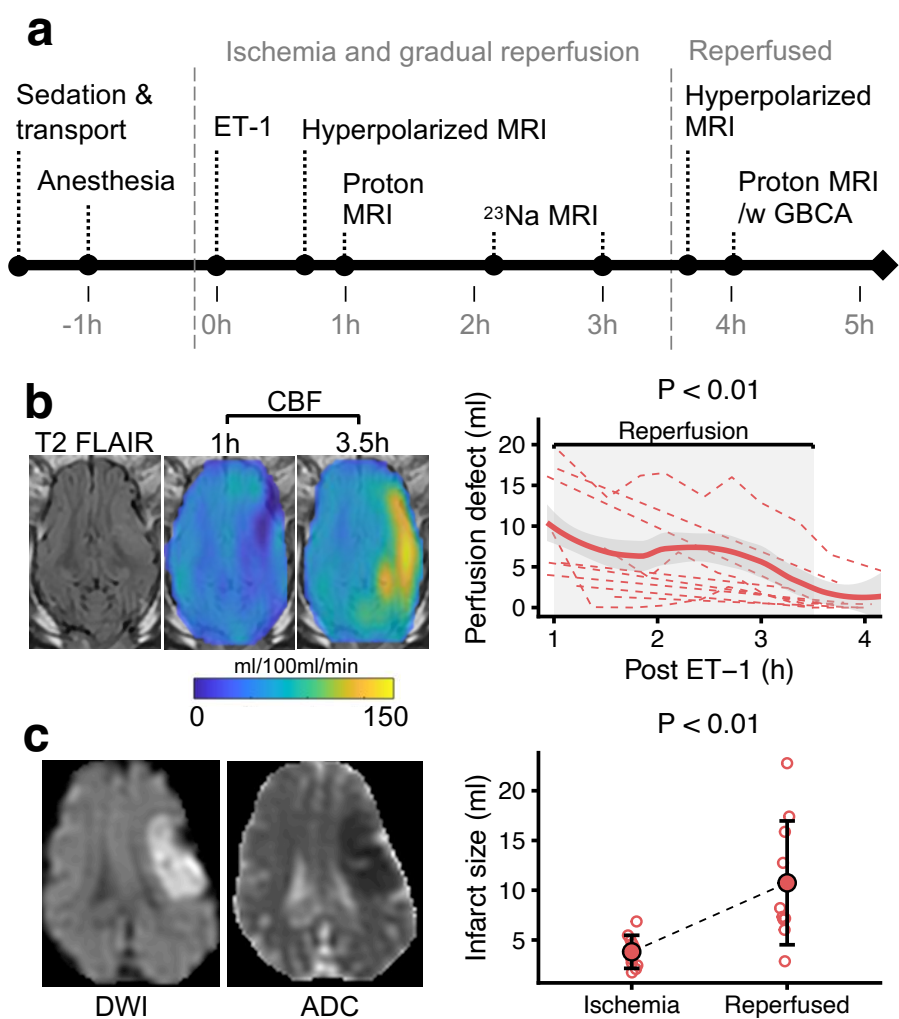

Figure 1: Intracerebral injection of endothelin-1 (ET-1) in pigs causes ischemia and infarction followed by gradual reperfusion. After intracerebral endothelin 1 (ET-1) injection, a magnetic resonance imaging (MRI) protocol was performed including hyperpolarized MRI (a, GBCA = gadolinium-based contrast agent). Cerebral blood flow (CBF) was quantified with arterial spin labeling MRI (b). The perfusion defect (CBF < $25 \mathrm{ml} / 100 \mathrm{ml} / \mathrm{min}$ ) shrank from 1 to 3.5 hours after ET-1. Diffusion weighted MRI (DWI) and apparent diffusion coefficient (ADC) maps showed a diffusion restriction 3.5 hours post ET-1 (c). The infarct (ADC < $\left.620 \times 10^{-6}\left[\mathrm{~mm}^{2} / \mathrm{s}\right]\right)$ grew from 1 hour (ischemia) to 3.5 hours (reperfused) after ET-1.

Data are shown as individual observations $(n=10)$ with mean \pm SD. In $c$, a local regression curve was added for visualization (thick line). Tested with linear mixed-effect models. 

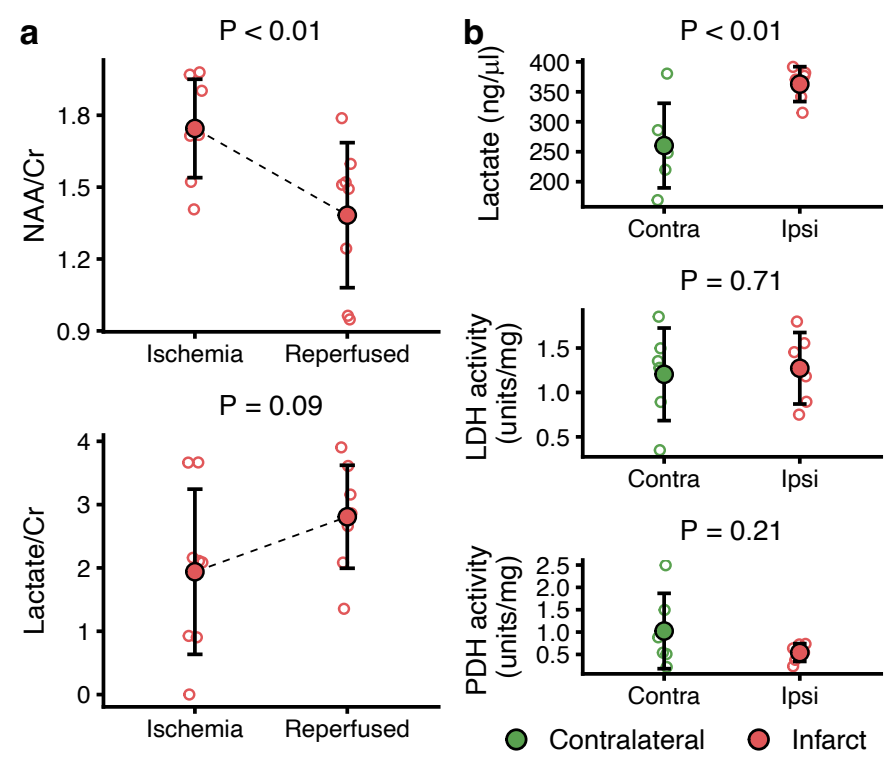

Figure 2: Characterization of metabolism in porcine ischemic stroke using magnetic resonance spectroscopy (MRS) and tissue samples. Determined with single-voxel ${ }^{1} \mathrm{H}$ MRS (a), N-acetylaspartate-tocreatine ratio $(\mathrm{NAA} / \mathrm{Cr})$ in the infarct decreased throughout the experiment, while lactate-to-creatine ( $\mathrm{Lac} / \mathrm{Cr}$ ) displayed a non-significant increasing tendency. After reperfusion and sacrifice, biopsies showed increased lactate concentration but no changes in normalized lactate dehydrogenase (LDH) or pyruvate dehydrogenase (PDH) activity in the infarcted ipsilateral compared to the contralateral brain (b).

Data $(n=6-8)$ are shown individual observations with mean \pm SD. Tested with linear mixed-effect models. 

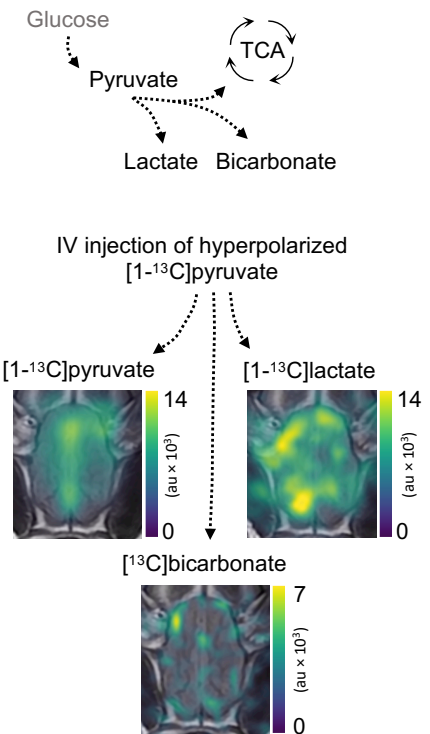

b

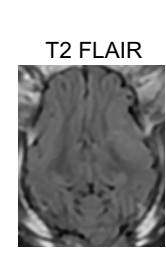

c

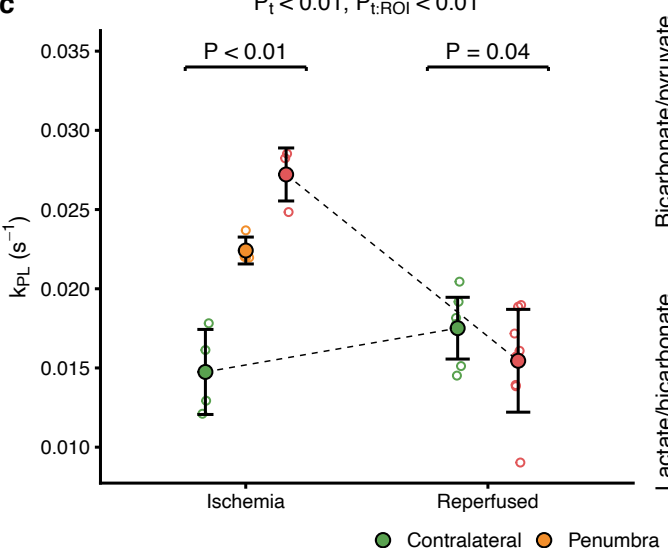

d $\quad \mathrm{P}_{\mathrm{t}}=0.71, \mathrm{P}_{\mathrm{t}: \mathrm{ROI}}<0.01$ 0.05

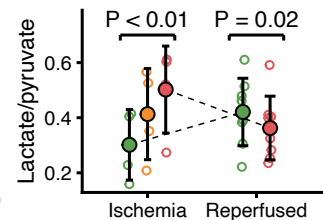

$\mathrm{P}_{\mathrm{t}}=0.33, \mathrm{P}_{\mathrm{t}: \mathrm{ROI}}<0.01$

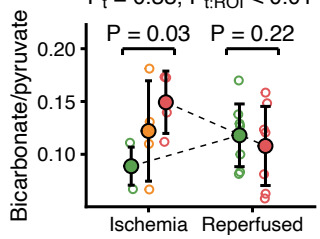

$P_{t}=0.4, P_{t: R O I}=0.8$

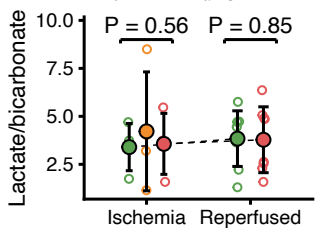

Figure 3: Hyperpolarized $\left[1-{ }^{13} C\right]$ pyruvate MRI detects the metabolic consequences of ischemic stroke and reperfusion in pigs. Hyperpolarized $\left[1-{ }^{13} \mathrm{C}\right]$ pyruvate was injected and the pyruvate and downstream metabolites were visualized with MRI ( $\mathrm{a}$, au = arbitrary unit). The anaerobic pyruvate-to-lactate exchange is sensitive to hypoxia. It was estimated through quantification of the rate constant $\left(k_{\mathrm{PL}}, \mathrm{b}+\mathrm{c}\right.$, red ring marks infarct) and using model-free ratio approaches (d) that showed increased lactate production during ischemia, which decreased to sub-normal levels after reperfusion.

Image examples are shown as one representative slice summed through time. Data are shown as individual observations ( $n=4-7)$ with mean \pm SD. Tested with linear mixed-effect models. ROI is region of interest, $\mathrm{t}$ is time, the colon (:) marks an interaction. 

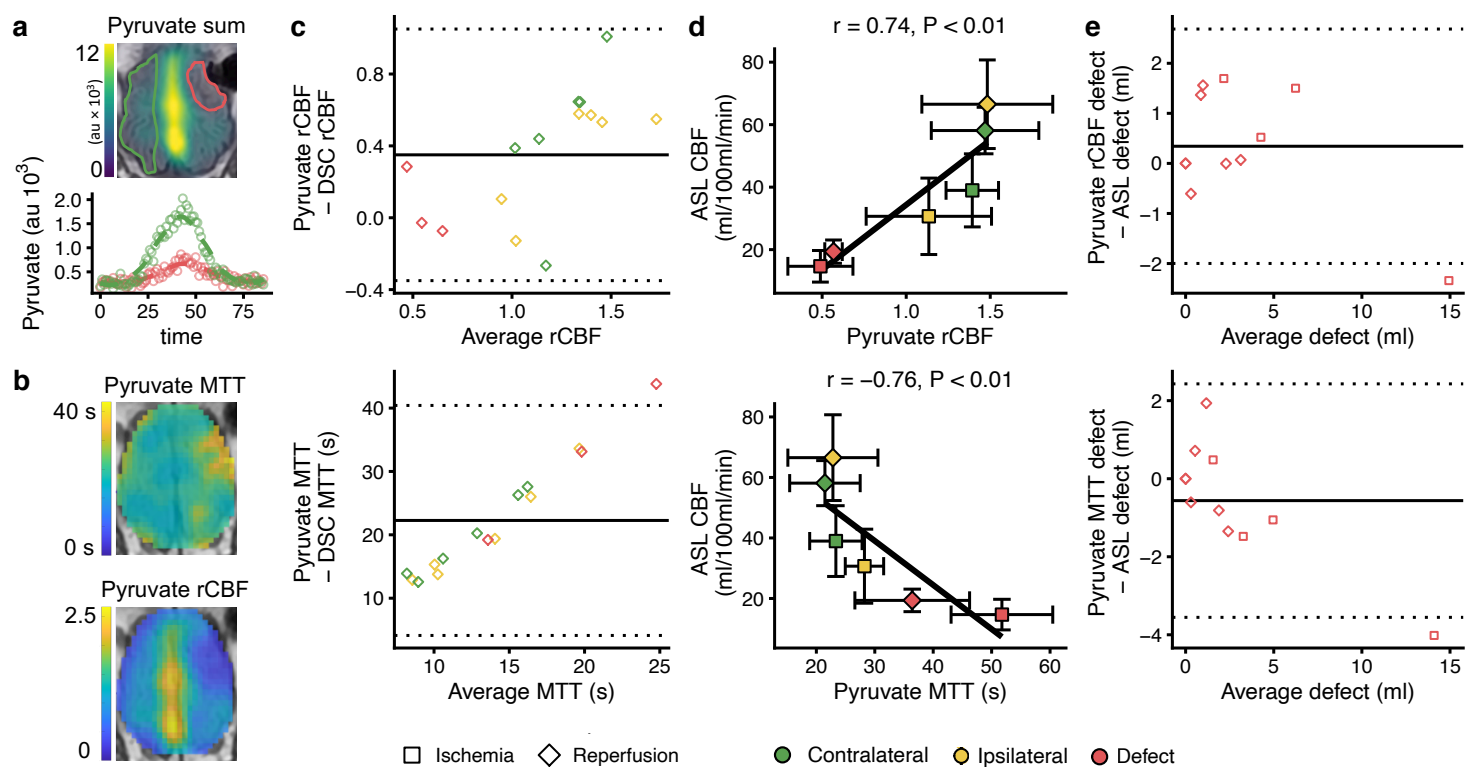

Figure 4: Perfusion-weighted imaging with hyperpolarized $\left[1-{ }^{13} C\right] p y r u v a t e$ shows the perfusion changes following endothelin-1 induced stroke in pigs. Delivery of hyperpolarized $\left[1-{ }^{13} \mathrm{C}\right]$ pyruvate was impaired during ischemia. A large signal was observed in the sagittal sinus ( $a$, au = arbitrary units). The time dynamics of the pyruvate signal were used to estimate perfusion via relative cerebral blood flow (rCBF) and mean transit time (MTT) following the principles of gadolinium-based dynamic susceptibility contrast (DSC) imaging (b). Bland-Altman plots showed that rCBF was slightly larger but unbiased compared with DSC, while MTT was much longer and skewed (c). The perfusion measures derived from the hyperpolarized pyruvate correlate well with CBF from arterial spin labeling MRI (ASL, d), and they showed good agreement with ASL in estimating size of the perfusion defect (e).

Data examples ( $a$ and $b$ ) are from one representative animal. Infarct and contralateral brain are marked with red and green, respectively. Data are shown as individual observations (c, e) or mean \pm SD (d). The curves were fitted as smooth local regressions (b) or linear regressions (d). As the same animals were 
examined during ischemia $(n=4)$ and after reperfusion $(n=7)$, the correlation analysis was performed under consideration of dependency in data. 


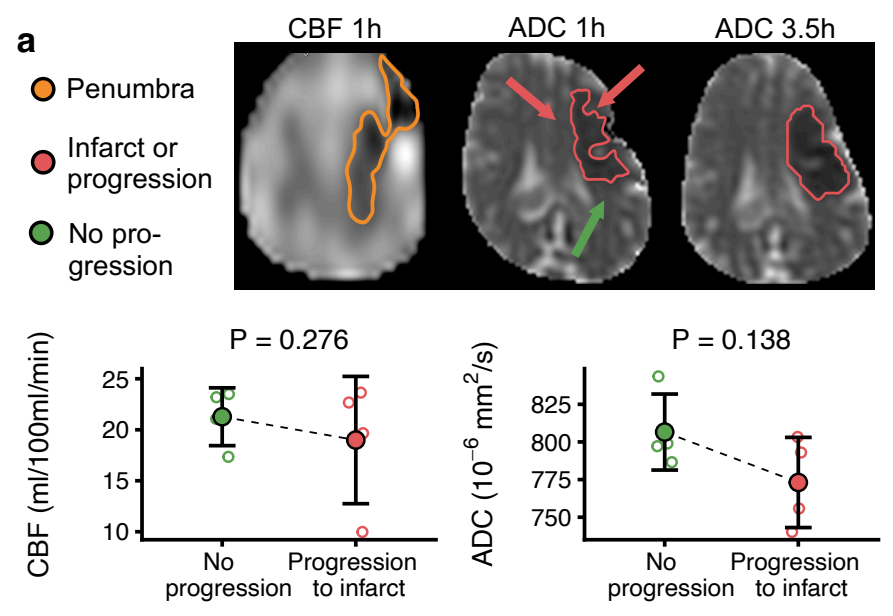

b $\quad k_{\mathrm{PL}} 1 \mathrm{~h} \quad$ Lac/pyr $1 \mathrm{~h} \quad$ Pyr rCBF $1 \mathrm{~h} \quad$ Pyr MTT $1 \mathrm{~h}$
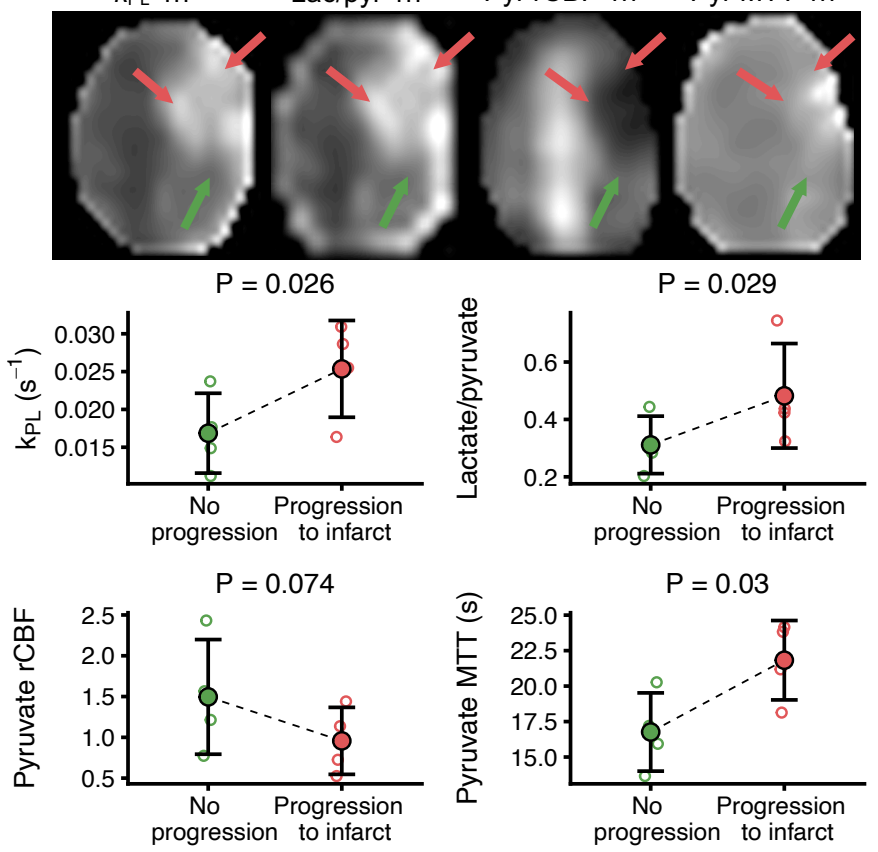

Figure 5: Hyperpolarized $\left[1-{ }^{13} \mathrm{C}\right]$ pyruvate $\mathrm{MRI}$ detects higher lactate production in the infarcting penumbra

in porcine stroke. From ischemia (1h) to the reperfused timepoint (3.5h), the infarct expanded into the penumbra. There were no differences in cerebral blood flow (CBF) measured with arterial spin labeling or apparent diffusion coefficient ( $A D C$ ) values between the progressing (red arrows) and the non-progressing 
parts (green arrow) of the penumbra (a). The parts of the penumbra that progressed to infarct displayed a higher rate of the pyruvate-to-lactate metabolism compared to the parts of the penumbra that did not (b). This effect was present in both model-based and model-free approaches. The relative CBF (rCBF) and mean transit time (MTT) measured with pyruvate perfusion suggested worse perfusion in the progressing regions.

Examples are from one representative animal. Data are shown as individual observations $(n=4)$ with mean \pm SD. Tested with linear mixed-effect models. 

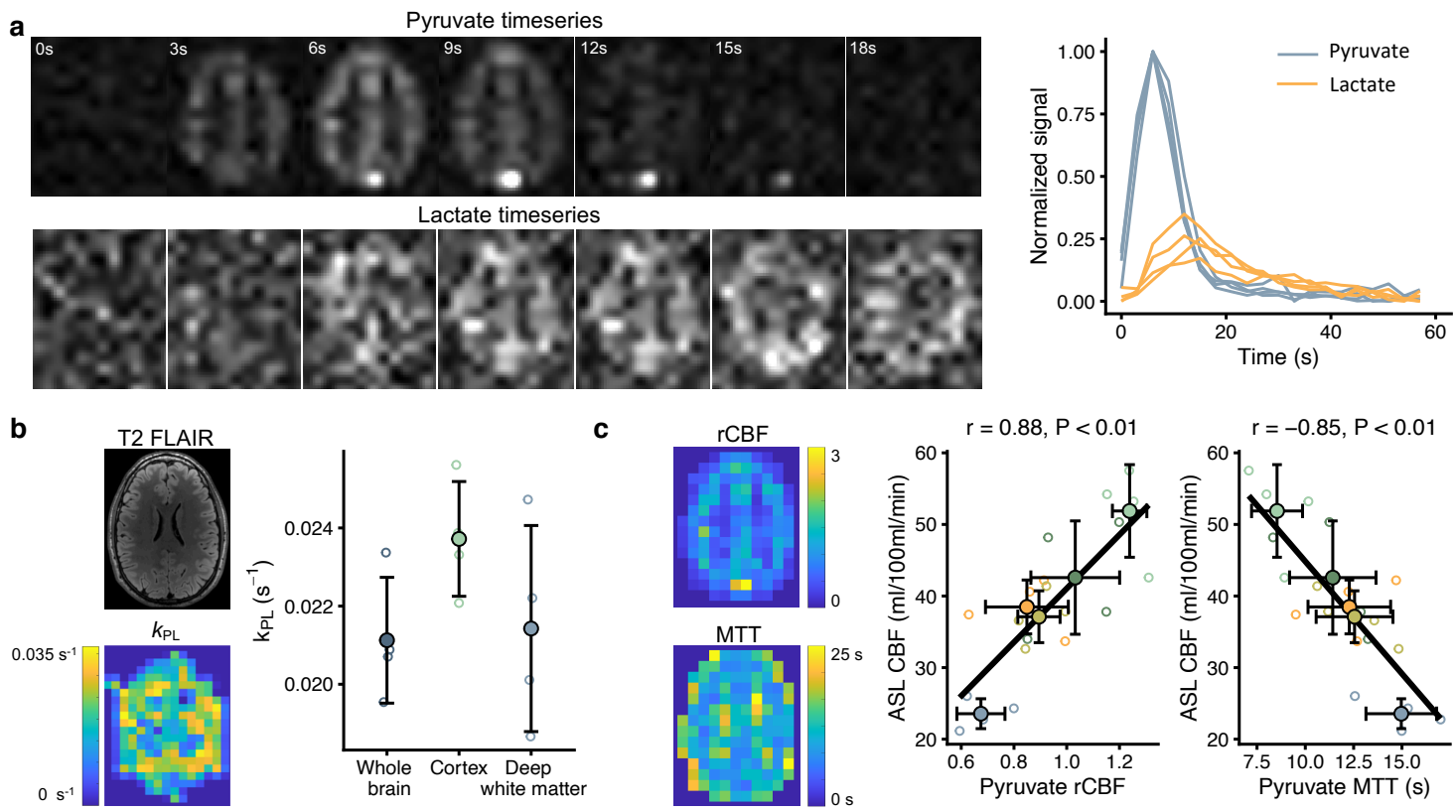

c
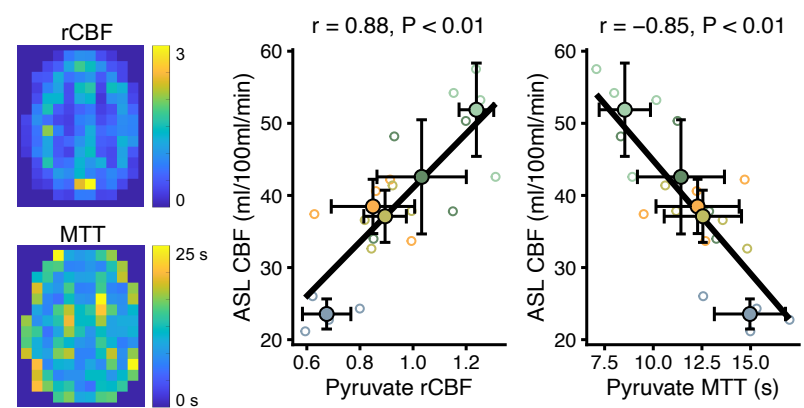

○ Cortex $\bigcirc$ Basal ganglia $\bigcirc$ Corpus callosum $\bigcirc$ Thalamus $\bigcirc$ Deep white matter

Figure 6: Hyperpolarized $\left[1-{ }^{13} \mathrm{C}\right]$ pyruvate $\mathrm{MRI}$ is translatable to concurrent studies of metabolism and perfusion in healthy humans. After injection of hyperpolarized pyruvate, pyruvate and lactate images were acquired every 3 seconds. A representative slice (first seven time points) is shown with time curves (normalized to maximal pyruvate signal) from all volunteers (a). From these, we modeled pyruvate-tolactate exchange $\left(k_{\mathrm{PL}}, \mathrm{b}\right)$. Further, we estimated perfusion $(\mathrm{c})$ through relative cerebral blood flow (rCBF) and mean transit time (MTT). Both showed good correlation to arterial spin labeling MRI (ASL).

Data are shown as individual observations $(n=4)$ with mean \pm SD. Correlation was assessed with repeatedmeasures analysis. 